\title{
On EXIT-Charts for Space-Time Block Codes
}

\author{
Aydin Sezgin ${ }^{1}$ \\ Heinrich-Hertz-Institut Berlin \\ Broadband Mobile Communication Networks \\ Einsteinufer 37, 10587 Berlin, Germany \\ e-mail: sezgin@hhi.de
}

\section{Wübben, R. Böhnke and V. Kühn \\ University of Bremen}

Department of Communications Engineering

Otto-Hahn-Allee 1, 28359 Bremen, Germany

e-mail: \{wuebben, boehnke, kuehn\}@ant. uni-bremen. de

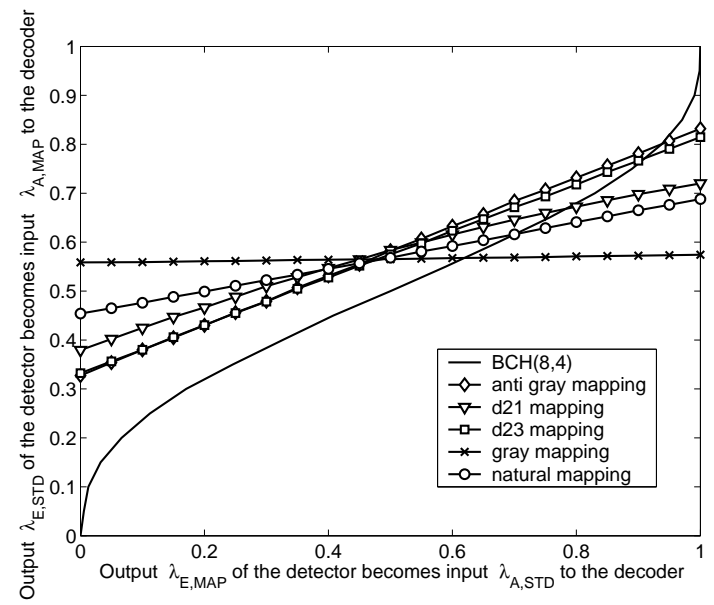

Figure 1: EXIT-chart of inner space-time-detector for $E_{b} / N_{0}=5 d B$. Additionally, the transfer characteristic for a rate $R_{\text {out }}=1 / 2$ outer code is depicted.

In the following, we analyze the impact of different 8PSK mappings (analogous to [1]) on the transfer characteristics of the detector for an AWGN channel. The extension to a Rayleigh fading channel is straightforward. With the chain rule of mutual information it can be shown that the mutual information $I(s ; r)=I(\mathbf{c} ; r)=I\left(c_{1}, \ldots, c_{m} ; r\right)$ between transmitted constellation symbol $s=f(\mathbf{c})$ and received AWGN channel output $r$ (assuming that all constellation symbols are equiprobable) can be decomposed into $I(\mathbf{c} ; r)=\sum_{L=0}^{m-1} I_{L}$, where $I_{L}$ is the average mutual information [1], when $L$ bits are already known to the receiver. Hence, for 8 PSK $(m=3)$ we have

$$
\begin{gathered}
I_{0}=\frac{1}{m\left(\begin{array}{c}
m-1 \\
0
\end{array}\right)} \sum_{i=1}^{m} I\left(c_{i} ; r\right) ; I_{1}=\frac{1}{m\left(\begin{array}{c}
m-1 \\
1
\end{array}\right)} \sum_{i=1}^{m} \sum_{\substack{j=1, j \neq i}}^{m} I\left(c_{i} ; r \mid c_{j}\right) ; \\
I_{2}=\frac{1}{m\left(\begin{array}{c}
m-1 \\
2
\end{array}\right)} \sum_{i=1}^{m} \sum_{\substack{j=1, i \\
j \neq i}}^{m} \sum_{\substack{k=j+1, k \neq i}}^{m} I\left(c_{i} ; r \mid c_{j}, c_{k}\right) .
\end{gathered}
$$

Note that conditioning (i.e. increasing a priori knowledge) increases the mutual information, i.e. $I_{L} \geq I_{L-1}$. In comparing (1) for different mappings, we observe that for Gray mapping the difference between $I_{2}$ and $I_{0}$ is not as large as in the case of the other mappings. This means that increasing a priori knowledge has only small impact on the information transfer of the detector for Gray mapping. Interestingly, this is not true for the other mappings, which we choose from all possible 8PSK mappings. Here, we observe an improved information transfer by increasing the a priori knowledge. Note that, $I_{0}$ is larger with Gray mapping in comparison to the other mappings. Therefore, we expect a better performance

\footnotetext{
${ }^{1}$ This work was supported in part by the German ministry of education and research (BMBF) under grant 01BU150 and grant $01 B U 153$.
}

with Gray mapping in the case, where no a priori knowledge is available at the receiver.

Next, we analyze the behaviour of the different mapping strategies with the help of EXIT-charts [2]. We consider a system with $n_{T}=2$ transmit and $n_{R}=1$ receive antennas. Fig. 1 shows the transfer characteristics of the space-time detector as the inner decoder for different mapping strategies. Different mappings result in transfer characteristics of different slopes. Note that the detector transfer characteristics are almost straight lines and increasing the SNR shifts the curve up. Additionally, note that for high SNR values, the slope of the detector transfer characteristics is also affected (not depicted here). From the figure, we see also that the natural mapping provides good extrinsic output at the beginning but provides diminishing output for higher a priori input $\lambda_{A}$. For the anti gray mapping it is the other way around. The detector which uses gray mapping provides almost the same extrinsic output for all a priori input. Therefore, we expect that the performance of the detector with gray and natural mapping is good in the low SNR regime and for a few iterations in comparison to the other mappings. But in the high SNR regime and for more iterations, we expect that it is the other way around.

\section{REFERENCES}

[1] S. ten Brink, "Designing iterative decoding schemes with the extrinsic information transfer chart," AEUE Intern. Journal of Electr. and Commun., vol. 54, pp. $187-219,2000$.

[2] S. ten Brink, "Convergence of iterative decoding," IEE Electron. Lett., vol. 35, no. 10, pp. 806-808, May 1999. 